Ann. Biol. anim. Bioch. Biophys., 1979, 19 (3 B), 915-927.

\title{
Rôle de la flore microbienne du tractus digestif chez le non-ruminant omnivore
}

\author{
par E. SACQUET
}

Laboratoire des animaux sans germes du C.N.R.S., I.N.R.A., 78350 Jouy-en-Josas.

Le rôle que joue la flore microbienne du tractus digestif dans les processus de digestion et d'absorption a fait l'objet de quatre revues récentes, dont l'une est en cours de publication (Wostmann, 1975 ; Combe ef al., 1976 ; Rérat, 1978 ; Coates, 1978). Le lecteur trouvera dans ces revues des informations détaillées ainsi qu'une abondante bibliographie.

Ce bref rapport introductif n'est pas une $5^{\mathrm{e}}$ revue des connaissances acquises dans ce domaine. Il a pour objet d'attirer l'attention sur des travaux récents ou sur certains points d'intérêt qui ne figurent pas dans les revues précédentes. Il se propose aussi d'être une étude critique de certaines recherches et de distinguer les directions dans lesquelles s'orientent les travaux ou dans lesquelles il serait sou haitable qu'ils s'orientent.

\section{Modifications de l'appareil digestif sous l'action de la flore microbienne}

L'une des principales idées qui se dégage de l'étude de l'organisme animal axénique, étude que nous appelons Gnotoxénie ou Gnotobiotique (le terme "Gnotobiotics » a été défini par Luckey, en 1963) est que l'organisme animal constitue avec la flore microbienne qui lui est associée un complexe. Les bactéries, étudiées in vitro, présentent souvent des caractéristiques très différentes de celles qu'elles possèdent dans le tractus digestif et il en est de même de l'organisme animal isolé des bactéries. C'est ainsi que la morphologie du tractus digestif, la vitesse de renouvellement de l'épithélium intestinal, la vitesse du transit gastro-intestinal, le débit sanguin au niveau des intestins, du foie, et secondairement l'ensemble de la circulation sanguine ainsi que le métabolisme de base sont modifiés chez le rongeur axénique, ef ces modifications retentissent évidemment sur la nutrition, sur la digestion et sur l'absorption.

Depuis la revue faite par Combe et al. (1976), quelques faits nouveaux ont été acquis dans ce domaine.

Pelkonen et Sickel (1978) remettent en cause certains résultats obtenus par les premiers expérimentateurs : le poids corporel du rat et celui de divers organes, par exemple, seraient moins différents en l'absence de germes que cela n'est habituelle- 
ment admis, parce que les premiers auteurs n'ont pas pris suffisamment de précautions pour qu'axéniques et holoxéniques soient génétiquement semblables et ne diffèrent que par un seul facteur: la présence ou l'absence de flore.

D'autres chercheurs essaient d'établir si les caractéristiques propres à l'état axénique, qui ont été le plus souvent étudiées chez une ou deux espèces de rongeurs, se retrouvent chez les autres espèces animales. C'est ainsi que le renouvellement de l'épithélium intestinal chez le rat, est, comme chez la souris plus lent à l'état axénique. Mais, bien qu'il s'agisse d'espèces voisines, ce ralentissement est plus faible chez le rat que chez la souris (Meslin et al., 1978). Rolls, Turvey et Coates (1978), viennent de montrer que, chez le poulet, qui à l'état axénique ne présente pas la distension caecale observée chez les rongeurs, le renouvellement de l'épithélium est plus lent en l'absence de flore microbienne. Bien que ces auteurs n'en fassent pas mention dans leur publication, les vitesses de renouvellement indiquées dans leurs travaux varient beaucoup selon le régime alimentaire que reçoivent leurs sujets d'expérience : la différence entre axénique et holoxénique apparaît plus importante avec un aliment complexe qu'avec un aliment semi-synthétique.

Dans toutes les espèces animales étudiées par Heneghan (1978), souris, rat, chien, porc, le nombre de cellules à mucus augmente en l'absence de flore microbienne, et la surface de l'épithélium intestinal tend à diminuer. Selon Baez ef al., 1978, Bruckner ef al., 1978, la réponse des vaisseaux sanguins et des muscles lisses de la paroi intestinale à l'action des catécholamines serait diminuée chez le porc axénique comme elle l'est chez le rat axénique.

D'après Dardillat (1978), des différences importantes de la motricité intestinale existent entre veau axénique et veau holoxénique.

Ainsi diverses caractéristiques de l'appareil digestif, d'abord observées chez les rongeurs axéniques, se retrouvent chez les autres espèces animales. Elles ne sont pas liées à la distension caecale observée chez les rongeurs et sont véritablement des caractères généraux de la vie sans germes.

Enfin, l'étude des mécanismes par lesquels la flore microbienne modifie l'appareil digestif, se poursuit. Chez le ral axénique, quand la bile est détournée de l'appareil digestif pendant un mois, le nombre des entérocytes au niveau des villosités iléales et la production cellulaire au niveau des cryptes de Lickerhhün deviennent semblables à ceux de l'holoxénique : c'est donc, au moins en partie, par les modifications qu'elle exerce sur les secrétions biliaires que la flore bactérienne accélère le renouvellement de l'épithélium iléal (Meslin ef al., 1978) chez le rat holoxénique.

L'isolement et l'identification des substances chimiques présentes dans le contenu intestinal des animaux axéniques, qui modifient la circulation sanguine ef la contraction de la fibre musculaire lisse de l'intestin n'ont pas fait de progrès importants depuis la revue de Combe ef al. (1976).

\section{Flore microbienne et digestion des matières azotées}

Les connaissances acquises dans ce domaine proviennent principalement de deux origines : la comparaison entre sujets axéniques et sujets holoxéniques ; la comparaison entre divers animaux holoxéniques qui reçoivent des aliments de composition 
différente ou sont soumis à des traitements antibiotiques. L'emploi de fistules iléales permet, chez le porc ou chez le chien de distinguer l'absorption au niveau de l'intestin grêle ef l'absorption au niveau de l'ensemble caecum-gros intestin.

L'ensemble de ces travaux est analysé d'une manière exhaustive par Rérat (1978), qui attire l'attention sur l'abondance des résultats contradictoires. Ces contradictions proviennent de ce que ces résultats ont été obtenus à l'aide d'espèces animales, de régimes alimentaires et de méthodes expérimentales différentes. C'est ainsi que le niveau de l'ingestion, la teneur en azote de la ration, la composition de celle-ci en matières azotées, et aussi en substances non azotées sont autant de facteurs susceptibles de modifier le résultat des expériences. Les récents travaux de Bewa (1978), Bewa et al. (1978) en fournissent un nouvel exemple : selon le type d'amidon utilisé (amidon type $A$ ou au contraire de type B), le taux de protéines de l'aliment (17 p. 100 ou 19 p. 100), la présence ou l'absence de 3 p. 100 de glucides solubles, la plus ou moins grande longueur de la chaîne de ces glucides, des différences spectaculaires de l'ingestion, de la croissance pondérale, de la rétention azotée et énergétique sont obtenues chez le poulet axénique et chez le poulet holoxénique.

Si on considère les méthodes utilisées, il apparaît que l'attention des chercheurs qui expérimentent uniquement à l'aide de sujets holoxéniques est particulièrement attirée par la perte en azote qui provient de l'incorporation de celui-ci dans les corps bactériens qui sont éliminés par les fèces. Ils utilisent le dosage de l'acide diaminopimélique pour apprécier la masse bactérienne excrétée par les fèces. Depuis que Levenson ef Tennant (1963) ont montré que le rat axénique, comparé au rat holoxénique, excrête davantage d'azote par la voie fécale, les chercheurs qui font de la Gnotobiotique le sujet de leur étude, envisagent des modes d'action de la flore bactérienne sur le métabolisme azoté qui sont très divers et qui ont été revus par Combe et al. (1976). L'action de la flore microbienne sur les matières azotées ne se limite pas à des actions cataboliques des bactéries et à la synthèse de leur tissu. Cette flore agit aussi en modifiant le renouvellement de l'épithélium intestinal. les synthèses enzymatiques de l'hôte, les mouvements des acides aminés à travers la paroi intestinale. Ces chercheurs s'intéressent non seulement aux variations quantitatives de l'excrétion fécale de bactéries mais aussi aux variations quantitatives de la flore et aux conséquences que celles-ci peuvent entraîner. II existe un certain nombre d'exemples dans lesquels il apparaît que des composants alimentaires modifient profondément les activités enzymatiques de la flore au niveau du cæcum et du gros intestin. Les études des métabolites bactériens des acides biliaires par exemple révèlent que l'introduction de 10 p. 100 de lactose dans l'aliment ou le remplacement d'amidon de maïs par de l'amidon cru de pomme de terre transforme la composition des fèces du rat en acides biliaires. Dans ce dernier cas, selon Kellogg (1971), la plupart des métabolites bactériens des acides biliaires disparaissent et la composition des fèces en acides biliaires se rapproche de celle des axéniques. Il est raisonnable de penser que des variations semblables des activités enzymatiques de la flore qui concernent d'autres métabolismes se produisent également. L'amidon cru de pomme de terre est d'ailleurs l'un des facteurs qui modifient la digestion des protéines alimentaires (Mason et Palmer, 1973). D'après Mendes Pereira, Pion et Prugnaud (1977) les glucides alimentaires modifient la synthèse protéique bactérienne chez le rat.

Si l'on considère les espèces animales de monogastriques omnivores, il est évident 
qu'elles diffèrent par de très nombreux caractères anatomiques et physiologiques, la composition de leur flore microbienne, la répartition de celle-ci dans les cavités digestives, leurs habitudes alimentaires, la pratique ou l'absence de pratique de la coprophagie. II n'est donc pas surprenant que les résultats des expérimentateurs diffèrent selon les espèces animales qu'ils ont étudiées.

Wostmann (1975) rappelle les travaux de Dubos et Schaedler (1960) qui, avec des régimes carencés en acides aminés essentiels, observent des croissances très différentes chez des souris qui diffèrent par la composition de leur flore microbienne, et diverses expériences qui tendent à montrer que le besoin en acides aminés, en lysine en parti-. culier, est moindre chez le rał ou la souris axénique (Gustafsson, 1967 ; Stoewsand ef al., 1968). D'après ces expériences la flore bactérienne des sujets holoxéniques joue un rôle nocif à l'égard de l'hôte puisqu'elle accroît le besoin en acides aminés, et l'importance de ce rôle nocif serait plus ou moins grand selon les flores intestinales.

Chez les espèces animales non coprophages, la présence de flore microbienne pourrait avoir un rôle défavorable lorsque les protéines alimentaires sont d'excellente qualité alors que, lorsque celles-ci sont altérées, par un traitement thermique par exemple, elle améliorerait la digestibilité de l'azote et parfois, sa rétention.Zebrowska, Buraczewska (1977) et Horaczynski observent, chez le porc holoxénique qui reçoit des protéines altérées, une amélioration de la rétention azotée sous l'action de la flore microbienne du cæcum et du gros intestin alors que Salter (1973) ne constate aucune amélioration chez le poulet holoxénique comparé au poulet axénique. Pour cet auteur, la flore microbienne, chez le poulet, agit uniquement par une action d'épargne de l'azote endogène. Cette action est très faible lorsque l'alimentation azotée est satisfaisante. Ce n'est que dans le cas de carence azotée très sévère que la flore microbienne, non seulement diminue la perte d'azote endogène, mais encore contribue à une meilleure utilisation des protéines alimentaires (Salter, Okumura et Coates, 1978). Dans les expériences de Bewa ef al. (1978) que nous avons précédemment mentionnées, la rétention azotée exprimée en p. 100 de l'ingéré varie de +25 p. 100 en faveur du poulet axénique, à - 18 p. 100 en sa défaveur, selon la composition de l'aliment. Ces variations s'accompagnent de variations de l'ingestion d'aliment ef sont probablement secondaires à celles- $\epsilon$ i. Mais cela n'enlève rien à l'intérêf nutritionnel de ces expériences, ef l'ampleur de ces variations de la rétention azotée sous l'action conjuguée de la flore et de l'aliment mérite d'être remarquée.

Il est très difficile, si l'on considère ces divers exemples de parvenir à une opinion satisfaisante sur la contribution qu'apporte la flore microbienne à la nutrition azotée de l'hôte. Pour Wostmann (1975) qui se limite aux expériences faites chez les rongeurs, la flore microbienne accroît le besoin de l'hôte en acides aminés. Coates (1978), dont les travaux portent sur le poulet, et Rérat (1978) qui analyse un très grand nombre de travaux, expriment une opinion semblable : pour ces auteurs, d'un point de vue nutritionnel, la digestion des matières azotées par la flore microbienne présente au niveau du tractus digestif n'est pas d'un grand profit pour les monogastriques omnivores.

Cette opinion est certainement exacte dans un grand nombre de cas, mais il reste à étudier si elle garde sa valeur lorsque l'apport azoté devient insuffisant, en particulier dans les conditions extrêmes de dénutrition ou de malnutrition. Un cerłain nombre de considérations invitent à poursuivre dans cette voie. Le rôle bénéfique de la flore bacté- 
rienne chez le poulet soumis à un régime déficient en protéine, observé par Salter et al. (1978), mérite d'être étudié chez d'autres espèces animales. Chez les espèces animales qui possèdent un cæcum ef un colon volumineux, l'action favorable ou nocive de la flore microbienne pourrait être plus importante que chez celles chez lesquelles ces organes sont peu développés et par conséquent plus importante chez le porc et chez l'homme que chez le rat, la souris ou le poulet. Le fait que certains facteurs alimentaires, même lorsqu'ils ne présentent qu'une faible fraction de l'aliment, produisent des modifications importantes de certaines activités enzymatiques de la flore bactérienne laisse penser qu'il est possible de modifier et peut-être d'orienter certaines de ces activités enzymatiques de la flore. Les expériences de Yang et al. (1972) selon lesquelles le rat est capable de digérer, d'absorber et d'incorporer une fraction appréciable des corps bactériens d'une culture d'E. coli méritent d'être poursuivies. En effet, parmi les différents processus selon lesquels les bactéries intestinales peuvent contribuer à la nutrition azotée de l'hôte, deux ont particulièrement retenu l'attention. Le premier est l'utilisation par l'organisme, pour la synthèse des acides aminés, de l'ammoniaque libéré par les désaminases bactériennes, et l'autre est la digestion des corps bactériens suivie de l'absorption et de l'utilisation des acides aminés d'origine bactérienne. Ce second processus présente certainement plus d'intérêt que le premier puisque l'utilisation métabolique des acides aminés synthétisés par l'organisme dans le premier processus est limitée, en théorie, par la quantité d'acides aminés essentiels disponibles, alors que de tels acides aminés existent dans les corps bactériens.

En conclusion, il nous semble qu'avant de poursuivre des études fines et approfondies sur les processus par lesquels la flore microbienne du tractus digestif modifie les aspects très divers du métabolisme azoté chez le monogastrique, il est nécessaire de quantifier cette action. II n'est pas douteux, si on prend en considération les travaux anciens faits chez les rongeurs ef les travaux plus récents de Salter (1978) et de Bewa et al. (1978) chez le poulet, qu'il existe des circonstances dans lesquelles le rôle de la flore microbienne devient important. II convient de définir ces circonstances. Une telle étude devrait être conduite en tenant compte de la multiplicité des facteurs susceptibles d'agir sur la relation flore microbienne-nutrition azotée, de la très grande diversité des conditions de milieu et d'alimentation dans lequel vivent l'homme et les diverses espèces animales, des conditions normales et pathologiques.

\section{Flore microbienne et digestion des glucides}

Il est classique de reconnaître que la flore microbienne du tractus digestif modifie la digestion des glucides et qu'elle intervient de multiples manières. Elle modifie la synthèse des enzymes de l'organisme-hôte : $p$. ex., les dissacharidases formées au niveau des entérocytes (Dahlqvist, Bull et Gustafsson, 1965 ; Reddy et Wostmann, 1966). Elle détruit ces enzymes, ex. : la maltase qui s'accumule dans le cæcum du rat axénique et est absente dans celui du rat holoxénique (Dahlquist, Bull et Gustafsson, 1965). Surtout, elle forme diverses hydrolases (cellulases, amylases, lactases, efc...) dont l'action est ensuite poursuivie jusqu'aux termes : acides gras volatils, $\mathrm{CO}^{2}, \mathrm{CH}^{4}, \mathrm{H}^{2}$. L'action de ces hydrolases bactériennes est d'autant plus grande que l'équipement enzymatique de l'hôte est souvent insuffisant pour assurer la digestion des matières glucidiques qu'il ingère. La digestion de la cellulose requiert la présence de bactéries 
spécifiques ; les bactéries intestinales jouent un rôle plus ou moins important dans la digestion de l'amidon selon l'espèce animale et le type d'amidon ; enfin la digestion de certains sucres, le lactose en particulier, chez l'adulte, est, pour une large part, le fait de lactases bactériennes.

Contrairement à ce qui concerne les substances azotées, il est reconnu que le rôle de la flore dans la digestion ef l'absorption des matières glucidiques est un processus d'une grande efficacité et d'un intérêt nutritionnel certain (Rérat, 1978). L'appréciation de sa valeur exacte se heurte certes à des difficultés techniques, en particulier parce qu'on ignore la quantité d'énergie qu'utilisent les bactéries pour leur synthèse, en sorte que dans les techniques où le critère de l'absorption est la disparition des matières énergétiques au niveau du cæcum et du gros intestin, l'énergie réellement absorbée ne peut être correctement mesurée. D'après Rérat (1978), la quantité de glucides ainsi digérée et absorbée représente chez un porc 5 à 20 p. 100 de la quantité ingérée. Elle varie évidemment sous l'influence de la substance considérée, fibres, cellulose, amidon, sucres divers, selon les autres éléments de la ration, selon l'espèce animale, etc...

Cette action de la flore bactérienne sur la digestion ef l'absorption des glucides n'est que l'un des aspects de la relation flore microbienne-glucides alimentaires. Le deuxième aspect est qu'inversement la composition glucidique de l'aliment modifie la flore bactérienne de l'hôte, ef que ces modifications de la flore retentissent sur divers métabolismes. Nous avons précédemment rappelé que la présence de lactose ou d'amidon de pomme de terre modifiait le métabolisme bactérien des acides biliaires chez le rat. L'introduction de lactose dans la ration réduit la digestion de la cellulose chez le porc (Février, Collet et Bourdon, 1973) ce qui ne peut s'expliquer que par une diminution des cellulases bactériennes. Il est très vraisemblable, bien que non démontré, que les variations de la concentration ef de la composition en acides gras volatils sous l'influence de la composition glucidique des aliments qui sont décrites dans la littérature et revues par Rérat (1978) sont le résultat de variations de la flore bactérienne du cæcum et du colon. Balmer et Zilversmit (1974), se demandent si une partie de l'action qu'exercent les fibres sur le métabolisme des sels biliaires et du cholestérol, ne relève pas de modifications de la flore bactérienne sous l'action des fibres, etc...

II est certain que la composition glucidique des aliments modifie la flore bactérienne du tractus digestif. Mais, cette action ainsi que les mécanismes par lesquels elle s'exerce, sont encore très mal connus. En particulier, le rôle des acides gras volatils, produits bactériens de la digestion des glucides, dans les interactions bactériennes n'est pas clairement démontré (Riottot, 1975). Parmi les multiples interactions bactériennes qu'ils ont éłudiées, Ducluzeau, Dubos et Raibaud (1971) ne donnent qu'un seul exemple où un lactobacille élimine du tractus digestif une autre bactérie, une Ristella, quand le sujet gnotoxénique consomme du lactose.

L'étude de la relation flore microbienne du tractus digestif-digestion et absorption des substances glucidiques constitue donc un vaste champ d'études où l'emploi d'animaux axéniques et gnotoxéniques semble s'imposer. Or, il est remarquable qu'elle fait actuellement l'objet de peu de travaux et que certains chercheurs n'en retiennent que les aspects négatifs. Coates (1978) remarque que « les mesures de l'énergie métabolisable de différents aliments donnés aux poulets en croissance ne donnent que des valeurs légèrement supérieures dans les conditions conventionnelles ». Aucun travail récent sur ce thème n'a été publié, à part celui de Bewa et al. (1978) qui, comme cela a 
déjà été mentionné, observent des différences importantes du niveau des ingesta, de la croissance pondérale, de la rétention azotée, mais aussi de la digestion de divers amidons et de la rétention énergétique, entre des poulets axéniques ef des poulets holoxéniques, qui reçoivent des aliments de composition différente, et en particulier des amidons de types différents.

Ces différences entre les résultats obtenus par divers chercheurs qui, cependant, expérimentent sur des sujets qui appartiennent à la même espèce animale, sont éloquentes. Elles illustrent combien il est nécessaire pour apprécier l'étendue et l'importance des relations entre la flore microbienne et la nutrition de l'hôte de réaliser des circonstances expérimentales très diverses.

\section{Flore microbienne et digestion des lipides}

Depuis la revue de Combe et al. (1976) une explication satisfaisante de la façon dont la flore microbienne du tractus digestif modifie l'absorption apparente des lipides alimentaires a été établie grâce aux travaux de Demarne et al. (1970, $1972 a, b)$, accomplis chez le rat axénique et holoxénique. L'ensemble de ces travaux peut être résumé comme suit. La présence d'une flore bactérienne dans le tractus digestif du rat a un effet défavorable sur l'absorption intestinale des matières grasses alimentaires. Cette action porte essentiellement sur les acides gras saturés longs (acides palmitique ef stéarique). Elle s'explique entièrement par le rôle qu'exerce la flore microbienne sur le métabolisme des sels biliaires. Chez le rat axénique, il y a deux à trois fois plus de sels biliaires au niveau de l'intestin que chez le rat holoxénique, Sacquet et al. (1975). II est possible en introduisant du taurocholate de sodium dans la ration d'élever chez le rat holoxénique le pool intestina! des sels biliaires au niveau que celui-ci atteint chez le rat axénique : la digestibilité des acides palmitique et stéarique devient alors chez ce rał holoxénique semblable à celle qui est observée chez le rat axénique (Demarne ef al., 1974). Le résultat de cette expérience s'accorde avec les connaissances précédemment établies sur la solubilisation micellaire des acides gras (Dawson et Webb, 1973 ; Fakambi, Flanzy et François, 1969 ; Flanzy, Rérat et François, 1968 ; Hoffman, 1970 ; Hoffman, Simmonds et Morgan, 1971 ; Hofmann, 1961, 1963). A concentration égale en sels biliaires conjugués, la solubilisation micellaire des acides gras longs et saturés est beaucoup plus faible que celle des acides gras longs insaturés. Une élévation de la concentration en sels biliaires au niveau de l'intestin grêle du rat provoquerait donc une augmentation de la solubilisation micellaire de ces acides gras longs saturés, mais modifierait peu celle des acides gras longs insaturés, qui est déjà excellente aux concentrations en sels biliaires observées chez l'holoxénique. Les acides gras saturés qui forment des micelles mixtes avec les sels biliaires conjugués et le 2-mono-acyl-glycerol seraient ainsi protégés de leur précipitation sous forme de savons calciques, et en conséquence, mieux absorbés.

La stéatorrhée qui est observée dans le syndrôme de l'anse aveugle porte au contraire sur les acides gras longs insaturés ef non pas sur les acides longs saturés (Demarne et al., 1971a, b). Dans ce syndrome les sels biliaires sont déconjugués au niveau de l'intestin grêle par les bactéries qui y prolifèrent en très grand nombre (Raibaud ef al., 1966 ; Sacquet ef al., 1966, 1968, 1971). Or, les sels biliaires déconjugués perdent leur propriété de former des micelles mixtes avec les acides gras (Hof- 
mann, 1961). Ainsi, la solubilisation micellaire des acides gras saturés qui est déjà mauvaise chez le sujet holoxénique normal, ne serait que faiblement affectée chez l'holoxénique atteint du syndrome de l'anse aveugle, alors qu'au contraire celle des acides gras insaturés, qui est excellente chez l'holoxénique normal serait diminuée dans une proportion importante chez l'holoxénique atteint du syndrome de l'anse aveugle.

Enfin, l'utilisation expérimentale de l'animal axénique et des méthodes récentes d'analyse des acides gras par chromatographie en phase gazeuse sur des colonnes capillaires permet de connaître la composition des fèces en acides gras d'origine endogène et de préciser ainsi notre connaissance des acides gras biosynthétisés par l'organisme animal (Demarne et al., 1978).

L'étude de l'action de la flore microbienne sur l'absorption et l'excrétion du cholestérol et des acides biliaires demeure très active. L'absence de transformation bactérienne chez l'axénique rend plus facile l'étude des produits de transformation du cholestérol.

Chez le rat axénique mâle, 35 p. 100 des produits de la transformation acide du cholestérol ne sont pas les acides biliaires classiquement décrits et ne sont pas dosés par les techniques classiques de chromatographie en phase gazeuse (Sacquet et al., 1978). II reste à démontrer dans quelle mesure la formation de ces substances inconnues est d'origine technique ou biologique.

Chez le rat mâle, il existe très peu d'acides biliaires sulfatés, moins de 2 p. 100 , alors que chez la femelle il en existe plus de 20 p. 100. Chez le mâle, les acides biliaires sont principalement les acides $\alpha$ - et $\beta$-muricholiques et cholique, alors que chez la femelle il existe, en plus de ces acides, de l'acide allochénodésoxycholique et allocholique (Eyssen et al., 1977). Chez la souris mâle la plus grande pariie de l'acide cholique est sulfaté en 7 (Eyssen, Parmentier et Mertens, 1976).

Le pourcentage de l'acide cholique par rapport à l'ensemble des acides biliaires biosynthétisés chez le rat mâle n'est pas modifié par la présence d'une flore microbienne (Sacquet et al., 1977a, b, c). Il diminue sous l'action de facteurs alimentaires : cholestérol (Gustafsson, Einarsson et Gustafsson, 1975 ; Sacquet et al., 1977a, b, c), lactose (Wostmann et al., 1976, 1977). Il est plus faible chez la femelle que chez le mâle (Einarsson, Gustafsson et Goldman, 1972 ; Eyssen ef al., 1977), ce qui s'observe aussi chez la souris (Eyssen ef al., 1973), et il évolue en fonction de l'âge (Eyssen et al., 1973).

Le pool intestinal et l'excrétion fécale des acides biliaires varient selon la composition de l'aliment, chez le rat axénique et chez le rat holoxénique (Wostmann ef al., 1976, 1977 ; Sacquet et al., 1977b). La comparaison entre rats axéniques, gnotoxéniques et holoxéniques permet de savoir si les facteurs alimentaires exercent leur action directement sur une propriété physiologique de l'organisme ou indirectement via la flore microbienne du tractus digestif. C'est ainsi qu'un aliment commercial, par comparaison avec un aliment semi-synthétique, élève le pool intestinal de sels biliaires, d'une valeur égale chez le rat axénique et chez le rat holoxénique : il exerce donc cet effet sans que la flore intervienne (Sacquef ef al., 1977b). Au contraire, les variations de l'excrétion fécale des acides biliaires sont principalement liées à des variations de la flore microbienne : un aliment de composition complexe, par comparaison à un aliment semisynthétique, accroît l'excrétion fécale des acides biliaires chez le rat holoxénique de façon significativement plus importante que chez le rat axénique : la flore microbienne est donc impliquée dans cette action (Sacquet et al., 1977b). 
La formation de certains métabolites bactériens des acides biliaires (acide $\omega$ muricholique par exemple) s'accompagne d'un accroissement de l'excrétion fécale des acides biliaires (Sacquet et al., 1977c).

Enfin, l'isolement de bactéries qui forment les différents métabolites observées chez les rats holoxéniques permet de tester la valeur des méthodes bactériologiques utilisées pour l'étude de la flore bactérienne du fractus digestif. L'étude des variations de la composition des fèces en acides biliaires constitue une méthode très fine d'analyse des variations de la flore bactérienne des cavités digestives sous l'action de divers facteurs, en particulier des facteurs alimentaires.

\section{Flore microbienne du tractus digestif et absorption et rétention des minéraux}

Depuis la revue de Combe et al. (1976), il n'existe à notre connaissance, dans ce domaine, aucun travail basé sur la comparaison entre axénique et holoxénique, à l'exception de celui de Andrieux, Guéguen et Sacquet (1978) qui étudient les effets du mode de stérilisation de l'aliment sur l'absorption ef la rétention des minéraux. II apparaît que, selon que l'aliment est stérilisé par autoclavage ou par irradiation, certaines caractéristiques de ce métabolisme sont modifiées et que ces modifications sont parfois différentes chez le rat axénique et chez l'holoxénique. La stérilisation des aliments constitue donc un artéfact en matière de métabolisme minéral, comme c'est le cas pour le métabolisme des protéines, des glucides ou des sels biliaires. La nécessité de stériliser les aliments des animaux sans germes peut conduire à des erreurs expérimentales, comme, par exemple dans le cas de Fe, dont l'absorption et la rétention sont plus faibles chez le rat axénique qui reçoit un aliment autoclavé que chez le rat holoxénique, alors que cette différence disparaît lorsque l'aliment est irradié.

\section{Flore microbienne et vitamines}

II n'existe, en Gnotobiotique, aucun travail récent concernant l'action de la flore microbienne sur le métabolisme vitaminique. Coates (1978) rappelle que la coprophagie est responsable, pour une large part, du profit que les rongeurs tirent des synthèses bactériennes de vitamines. Comme la plupart des travaux ont été accomplis chez les rongeurs, qui sont coprophages, et chez le poulet qui possède un intestin terminal relativement peu développé par rapport à celui des autres monogastriques omnivores et en particulier par rapport à celui de l'homme, le profit que tirent ces dernières espèces animales des synthèses bactériennes demeure indéterminé.

\section{Conclusion}

Cette étude critique est incomplète, puisqu'elle ne traite ni de la digestion bactérienne des substances médicamenteuses ou toxiques, malgré l'importance croissante qu'on lui reconnaît (Goldman, 1978), ni de l'action de la flore bactérienne sur la digestion des aliments dans les conditions pathologiques. Le rôle de cette flore dans les conditions physiologiques est encore imparfaitement connue ef les récentes revues qui 
en ont été faites, font état de résultats expérimentaux ef d'opinions contradictoires dans de nombreux domaines. Cet état des connaissances s'explique sans doute par la complexité des phénomènes étudiés : l'appareil digestif et la flore microbienne qui lui est associée, constitue un système d'interactions multiples soumis aux influences extérieures parmi lesquelles celle du régime alimentaire est sans doute, l'une des plus importantes. Les facteurs alimentaires peuvent exercer leur action, soif directement sur l'organisme, soit indirectement en provoquant des modifications de la flore qui retentissent à leur tour sur les processus de digestion, d'absorption, d'excrétion. La complexité de ces phénomènes soumet l'expérimentateur à deux exigences qui sont difficiles à concilier :

- d'une part : faire des expériences très précises, dans lesquelles tous les facteurs de variation sont définis, parce qu'une variation d'un facteur est susceptible d'avoir des conséquences multiples et imprévisibles ;

- d'autre part : réaliser au contraire des conditions expérimentales très diverses, faute de quoi il lui est impossible d'apprécier l'action de la flore microbienne dans toute sa diversité.

Commission CNERNA Digestion-Absorption/Association des Physiologistes, Paris 5-6 octobre 1978.

\section{Références}

ANDRIEUX C., GUÉGUEN L., SACQUET E., 1978. Effects of the mode of sterilization of diet on mineral absorption in germ-free and conventional rats. 6th int. Symp. Gnotobiotics. June 1978, Ulm/Donau, Fed. Rep. Germany.

BAEZ S., BRUCKNER G., WALDEMAR Y. P., MINIATS O. P., GORDON H. A., 1978. Vascular smooth muscle depressant substance in germ-free piglets. 6th. int. Symp. Gnotobiotics. June 1978, Ulm/Donau, Fed. Rep. Germany.

BALMER J., ZILVERSMIT D. B., 1974. Effects of dietary roughage on cholesterol absorption, cholesterol turnover and steroid excretion in the rat. J. Nutr., 104, 1319-1328.

BEWA H., 1978. Amidon des tubercules tropicaux. Efficacité nutritionnelle pour le poulet. Thèse Dr-Ing., Paris.

BEWA H., CHARLET G., FISZLEWICZ M., LE COZ Y., MOREL M. T., SZYLIT O., 1978. Influence of the axenic state on the utilization of carbohydrates by chicken. 6th. int. Symp. Gnotobiotics, June 1978, Ulm/Donau, Fed. Rep. Germany.

BRUCKNER G., BAEZ S., MINIATS O. P., GORDON H. A., 1978. Intestinal and vascular autonomic sensitivity of germ-free and conventional piglets. $6 \mathrm{th}$ int. Symp. Gnotobiotics. June 1978, Ulm/ Donau, Fed. Rep. Germany.

COATES M. E., 1978. Nutrition and metabolism in the gnotobiotic state. 6th int. Symp. Gnotobiotics. June 1978, Uim/Donau, Fed. Rep. Germany.

COMBE E., DEMARNE Y., GUÉGUEN L., IVOREC-SZYLITT O., MESLIN J. C., SACQUET E., 1976. Some aspects of the relationships between gastrointestinal flora and host nutrition. WId. Rev. Nutr. Diet, 24, 1-57.

DAHLOVIST A., BULL B., GUSTAFSSON B. E., 1965. Rat intestinal 6-bromo, 2-naphtyl glycosidase and dissacharidase activities. I. Enzymatic properties and distribution in the digestive tract of conventional and germ-free animals. Arch. Biochem. Biophys., 109, 150-158.

DARDILLAT C., 1978. (données non publiées).

DAWSON A. M., WEBB, J. P. W., 1973. - Oleic acid absorption from micellar solutions and emulsions in the rat. Proc. soc. exp. Biol., 142, 906-908.

DEMARNE Y., SACQUET E., FLANZY J., GARNIER H., FRANÇOIS A. C., 1970 . Utilisation digestive apparente des acides gras chez le rat axénique et holoxénique. Ann. Biol. anim. Bioch. Biophs., 10, 369-384.

DEMARNE Y., SACQUET E., FLANZY J., GARNIER J., FRANÇOIS A. C., 1971o. Type de matière 
grasse et stéatorrhée. 1) Effeł de l'ingestion d'une matière grasse saturée. Ann. Biol. anim. Bioch. Biophys., 11, 725-727.

DEMARNE Y., SACQUET E., FLANZY J., GARNIER H., FRANÇOIS A. C., 1971b. Type de matière grasse et stéatorrhée. 2) Effet de l'ingestion d'huile de colza. Ann. Biol. anim. Bioch. Bioph., 11, 729-731.

DEMARNE Y., SACQUET E., FLANZY J., 1972a. The influence of gastro-intestinal flora on digestive utilization of fatty acids in rat, 553-560. In HENEGHAN J. B., Germ-free research. Biological effects of Gnotobiotic environments. Acad. Press, N. Y.

DEMARNE Y., SACQUET E., FLANZY J., GARNIER H., 1972b. Influence cumulée de la caecectomie et de l'éfat axénique sur l'utilisation digestive apparente de la ration et des acides gras chez le rat. Ann. Biol. anim. Bioch. Biophys., 12, 139-148.

DEMARNE Y., SACQUET E., VAN HEIJENOORT Y., MATHIS C., 1974. Influence d'une supplémenfation de la ration en sels biliaires conjugués sur l'équipement en acides biliaires de l'intestin grêle du rat. Modifications de l'absorption apparente des acides gras. Ann. Biol. anim. Bioch. Biophys., 14, 239-250.

DEMARNE Y., 1976. Influence de la flore du tractus digestif sur les mécanismes de digestion ef d'absorption des lipides alimentaires. Coniribution à l'éfude du phénomène chez le rat. Thèse Doct.-Ing., Univ. Nancy I.

DEMARNE Y., SACQUET E., LECOURTIER M. J., FLANZY J., 1978. Comparative study of endogenous fecal fatty acids in germ-free and conventional rats. Am. J. Clin. Nutr., (à paraître).

DUBOS R. J., SCHAEDLER R. W., 1960. The effect of intestinal flord on the growth rate of mice and on their susceptiblity to experimental infection. J. exp. Med., 111, 407.

DUCLUZEAU R., DUBOS R., RAIBAUD P., 1971. Effet antagoniste d'une souche de Lactobacillus sur une souche de Ristella sp., dans le tube digestif de souris "gnotoxénique » absorbant du lactose. Ann. Inst. Pasteur, 121, 777-794.

EINARSSON K., GUSTAFSSON J. A., GOLDMAN A. S., 1972. Metabolism of steroid hormones, sterols and bile acids in liver microsomes from male, female and pseudohermaphrodic rats. Eur. J. Biochem., 31, 345-353.

EYSSEN H., PARMENTIER G., MERTENS J., DE SOMER P., 1973. The bile acids of the mouse : effect of microflora, age and sex, 271-276. In HENEGHAN J., Germ-free research. Aca-. Press, New York, London.

EYSSEN H., PARMENTIER G., MERTENS J., 1976. Sulfated bile acids in germ-free and conventional mice. Eur. J. Biochem., 66, 507-514.

EYSSEN H. SMETS L., PARMENTIER G., JANSSEN G., 1977. Sex-linked differences in bile aciJ metabolism of germ-free rats. Life Sci., 21, 707-712.

FAKAMBI L., FLANZY J., FRANÇOIS A. C., 1969. Competition in vivo entre acides gras et phosphore pour la formation de composés insolubles. C. R. Acad. Sci. Paris, Série D, 269, 2233-2235.

FÉVRIER C., COLLET J., BOURDON D., 1973. Utilisation de divers types de lactosérum dans les régimes de sevrage des porcelets et durant la période de croissance finition. Journées Rech. Porcine en France, Paris, I.N.R.A.-I.T.P. Ed., 79.

FLANZY J., RERAT A., FRANÇOIS A. C., 1968. Etude de l'utilisation digestive des acides gras chez le porc. Ann. Biol. anim. Bioch. Biophys., 8, 537-548.

GOLDMAN P., 1978. Biochemical pharmacology of the intestinal flora. Ann. Rev. Parmacol. Toxicol., 18, 523-539.

GUSTAFSSON B. E., 1967. Introduction of specific microorganisms into germ-free animals, 16. In Nutrition and infection Ciba Study Group n ${ }^{\circ} 31$, Little Brown Co, Boston.

GUSTAFSSON B. E., EINARSSON K., GUSTAFSSON J. A., 1975. Influence of cholesterol feeding on liver microsomal metabolism of steroids and bile acids in conventional and germ-free rats. J. biol. Chem., 250, 8496-8502.

HENEGHAN J. B., 1978. Enterocyte kinetics, mucosal surface area, and goblet cells in gnotobiotics. 6th int. Symp. Gnotobiotics. June 1978, Ulm/Donau, Fed. Rep Germany.

HOFFMAN N. E., 1970. The relationships between uptake in vitro of oleic acid and micellar solubilization. Bioch. biophys. Acta, 196, 193-203.

HOFFMAN N. E., SIMMONDS W. J., MORGAN R. G. H., 1971. A comparison of absorption of free fatty acids and $\alpha$-glycerol ether in the presence and absence of micellar phase. Bioch. biophys. Acta, 231, 487-495. 
HOFMANN A. F., 1961. Micellar solubilization of fatty acids and monoglycerides in bile salt solution, 159-171. In DESNUELLE P., The enzymes of lipid mefabolism. Pergamon Press Oxford, London, New York.

HOFMANN A. F., 1963. Further studies on the intraluminal phase of fat digestion factors influencing encing the behaviour of fatty acids in bile salt solution, 501. Résumé des Commun. 6e Congr. mond. Nutr. Edinbourg.

KELLOG T. F., 1971. Microbiological aspects of enterohepatic neutral sterols and bile acid metabolism. Fed. Proc., 30, 1808-1814.

LEVENSON S. M., TENNANT B., 1963. Some metabolic and nutritional studies with germ-free animals. Fed. Proc., 22, 109-119.

LUCKEY T. D., 1963. Germ-free life and gnotobiology. Acad. Press, N. Y., and London, pp. 487.

MASON V. C., PALMER R., 1973. The Influence of bacterial activity in the alimentary canal of rats on faecal nitrogen excretion. Aclo agr. scand., 23, 141.

MENDES PEREIRA E., PION R., PRUGNAUD J., 1977. Utilisction digestive apparente des acides aminés de quelques aliments chez le rat en croissance. Ann. Biol. anim. Bioch. Biophys., 17, 625-631.

MESLIN J. C., SACQUET E., RIOTTOT M., LEGLISE P. C., 1978. Effet d'une dérivation de la bile hors du tractus digestif pendant une période prolongée, sur le renouvellement de l'épithélium iléal chez le rat axénique et le rat holoxénique. Ann. Biol. anim. Bioch. Biophys., 18, 295. 303.

PELKONEN K. H. O., SICKEL E., 1978. Morphological characterization of the outbred adult germfree and conventional Ham Wistar rat. 6th int. Symp. Gnotobiotics. June 1978, Ulm/Donau. Fed. Rep. Germany.

RAIBAUD P., DICKINSON A. B., SACQUET E., CHARLIER H., MOCQUOT J., 1966. La microflore du tube digestif du rat. Ann. Inst. Pasteur, 111, 193-210.

REDDY B. S., WOSTMANN B. S., 1966. Intestinal dissacharidases activities in the growing germfree and conventional rats. Arch Biochem. Biophys., 113, 609-616.

RÉRAT A., 1978. Digestion and absorption of carbohydrates and nitrogenous matters in the hindgut of the omnivorous non-ruminant animal. J. Anim. Sci., 46, 1808-1837.

RIOTTOT M., 1975. Production d'acides gras volotils dans le cœecum de la souris. Effets sur Shigella Flexneri chez cet animal. Thèse $3^{\mathrm{e}}$ cycle, Paris 7.

ROLLS B. A., TURVEY A., COATES M. E., 1978. The influence of the gut microflora and of dietary fibre on epithelial cell migration in the chick infestine. Br. J. Nutr., 39, 91-98.

SACQUET E., CHARLIER H., RAIBAUD P., DICKINSON A. B., EVRARD E., EYSSEN H., $1966 . \quad$ Etiologie bactérienne de la stéatorrhée observée chez le rat porteur d'un cul-de-sac intestinal. C. R. Acad. Sci., Paris, Série D., 262, 786-789.

SACQUET E., GARNIER H., RAIBAUD P., EYSSEN H., 1968. Ełiologie bactérienne de la stéatorrhée observée chez le rat porteur d'un cul-de-sac intestinal. Déconjugaison de l'acide taurocholique. C. R. Acod. Sci., Paris, Série D, 267, 2238-2240.

SACQUET E., 1971. Flore microbienne du tractus digestif et nutrition chez Rattus norvegicus. Thèse Doct. Etat Sci. nat., Fac. Sci. Univ. de Paris.

SACQUET E., VAN HEIJENOORT Y., RIOTTOT M., LEPRINCE C., 1975. Action de la flore microbienne du tractus digestif sur le métabolisme des acides biliaires chez le rat. Biochim. biophys. Acta, 380, 52-65.

SACQUET E., MEJEAN C., LEPRINCE C., RIOTTOT M., 1977a. Action de la flore microbienne du tractus digestif sur la biosynthèse de l'acide cholique chez le rat. Biochim. biophys. Acta, 499, 67-72.

SACQUET E., MEJEAN C., LEPRINCE C., RIOTTOT M., RAIBAUD P., 1977b. Action du régime alimentaire ef de la flore microbienne du tractus digestif sur le pool intestinal et l'excrétion fécale des acides biliaires chez le rat : étude comparée chez des rats axéniques, gnotoxénique ef holoxéniques. Ann. Nutr. Alim. 30, 603-617.

SACQUET E., LEPRINCE C., RIOTTOT M., MEJEAN C, LEGLISE P., 1977c. Formation d'acide $\omega$-muricholique et excrétion fécale des acides biliaires chez le rat. C. R. Acad. Sci., Paris, Sér. D., 284, 557-559.

SACQUET E., LEPRINCE C., RIOTTOT M., MEJEAN C., RAIBAUD P., 1978. Measurement of fecal bile acid excretion in gnotobiotic rats : comparison of gas-liquid chromatography and ${ }^{4-14} \mathrm{C}$ cholesterol isotopic equilibrium. Steroids, 32, 1-12. 
SALTER D. N., 1973. The influence of gut micro-organisms on utilization of diefary protein. Proc. Nutr. Soc., 32, 65-71.

SALTER D. N., OKUMURA J., COATES M. E., 1978. Nitrogen conservation by the gut microflora. 6th int. Symp. Gnotobiotics. Juin 1978, Ulm/Donau. Fed. Rep. Germany.

STOEWSAND G. S., DYMSZA H. A., AMENT D., TREXLER P. C., 1968. Lysine requirement of the growing gnotobiotic mice. Life Sci., 7, 689.

VAN HEIJENOORT Y., SACQUET E., RAIBAUD P., DEMARNE Y., MATHIS C., 1972. Action de la flore microbienne sur le contenu de l'intestin grêle en acides biliaires. C. R. Acad. Sci., Paris, Série $D, 275,271-274$.

WOSTMANN B. S., 1975. Nutrition and metabolism of the germ-free mammal. WId. Rev. Nutr. Diet., 22, 40-92.

WOSTMANN B. S., BRUCKNERR-KARDOSS E., BEAVER M., CHANG L., MADSEN D., $1976 . \quad$ Effect of dietary lactose at levels comparable to human consumption on cholesterol and bile acid metabolism of conventional and germ-free rats. J. Nutr., 106, 1782-1790.

WOSTMANN, B. S., BEAVER M., CHANG L., MADSEN D., 1977. Effect of autoclaving of a lactosecontaining diet on cholesterol and bile acid metabolism of conventional and germ-free rats. Am. J. clin. Nut., 30, 1999-2005.

YANG M. G., BERGEN W. G., SCULTHORPE A. E., MICKELSEN O., 1972. Utilization of ${ }^{14} \mathrm{C}$ labeled. E. coli by the rat caecum and after forcefeeding. Proc. Soc. exp. Biol. Med., 139, 1312.

ZEBROWSKA T., BURACZEWSKA L., HORACZYNSKI H., 1977. Apparent digestibility of nitrogen and aminoacids and utilization of protein given per os or to the caecum in pigs. 5 th int. Symp. Aminoacids Budapest, Feb. 1977 (cité par Rérat, 1978). 